\title{
Eigen-Beamforming with Delayed Feedback and Channel Prediction
}

\author{
T. R. Ramya and Srikrishna Bhashyam \\ Department of Electrical Engineering, \\ IIT Madras, Chennai - 600 036, India. \\ Email: \{ee04d016,skrishna\}@ee.iitm.ac.in
}

\begin{abstract}
Adaptive transmit beamforming based on channel state information (CSI) is a key feature in next generation wireless cellular systems. However, CSI available for adaptation is imperfect due to feedback delay and estimation errors. In this work, we analyze the outage performance of maximum eigen-mode beamforming with imperfect CSI. First we analyze the outage probability in terms of the correlation coefficient $\rho$ between the CSI available at the transmitter (CSIT) and the CSI available at the receiver (CSIR). The analysis shows that feedback delay leads to significant degradation at medium and high signal-to-noise ratios (SNR). Furthermore, the effect of delay can be overcome only if $\rho$ tends to one with increasing SNR. Then, we study whether linear minimum mean squared error (MMSE) prediction can achieve the required behavior in $\rho$. The length of the prediction filter required is numerically evaluated and shown to increase with SNR. Finally, the asymptotic diversity order is analyzed as a function of the rate at which $1-\rho$ approaches 0 as the SNR $\rightarrow \infty$. Results show that for $1-\rho$ proportional to $S N R^{-1}$, the asymptotic diversity order remains unaltered.
\end{abstract}

\section{INTRODUCTION}

Adaptive Multiple Input Multiple Output (MIMO) transmission based on the Channel State Information (CSI) is a key feature incorporated in next generation cellular systems like 3GPP LTE and 3GPP LTE-advanced [1] to improve performance. In Frequency Division Duplex (FDD) systems, the channel is estimated at the receiver using training symbols and fed back to the transmitter. In Time Division Duplex (TDD) systems, training symbols can be used in both links to estimate the channel state, assuming channel reciprocity. Codebook-based and non-codebook-based transmit beamforming are important schemes proposed in the class of adaptive MIMO schemes. With CSIT, the MIMO channel can be split into multiple parallel subchannels using singular value decomposition (SVD). For transmitting a single data stream, maximum eigen-mode beamforming that uses only the channel corresponding to the maximum singular value is optimal in terms of received SNR [2,3] and achieves the maximum diversity gain. Maximum eigen-mode beamforming is a good candidate for non-codebook-based beamforming.

In practice, perfect CSIT is not possible due to channel estimation errors and feedback delay. The effect of feedback delay on closed-loop transmit diversity was studied in [4] and the effect of imperfect CSIT on the outage performance of Multiple Input Single Output (MISO) beamforming was analyzed in [5]. The capacity gain of a transmit beamforming system compared to the case of no feedback is shown to decrease at least exponentially with feedback delay in [6]. The effect of feedback delay and estimation error on MIMO multiplexing systems based on the eigen-beams obtained using singular value decomposition (SVD) are studied in [7-10]. In [7] and [8], feedback delay is shown to result in interference between the eigen-beams. While zero-forcing (ZF) and MMSE receivers are proposed in [7] to compensate for the effect of feedback delay, prediction is proposed and compared with $\mathrm{ZF}$ and MMSE methods in [8] using simulations. In $[9,10]$, the bit error rate and outage probability are evaluated for constellations with finite number of symbols assuming that the interference between channels is Gaussian. Since all eigenmodes are used in [7-10], the performance is limited by the worst eigen-mode even with perfect CSI. Furthermore, the interference between modes leads to error floors. The effect of delay on a single beam is dominated by the interference from the beams. In [3], maximum eigen-mode beamforming is studied and the use of prediction is evaluated in the context of adaptive modulation using uncoded rectangular QAM constellations with a target bit error rate. However, channel estimation errors are neglected in [3].

In this paper, we analyze the outage probability of the MIMO maximum eigen-mode beamforming in the presence of imperfect CSI in terms of the correlation coefficient $\rho$ between the CSIT and the CSIR. We first show that the degradation due to feedback delay is significant at medium and high SNR. In comparison, the effect of channel estimation error is not significant at high SNR because the estimation error decreases with SNR. Then, we study whether prediction can compensate the effect of feedback delay. This requires the prediction filter length to increase with SNR. Finally, the asymptotic diversity order is analyzed as a function of the rate $\alpha$ at which $\rho$ approaches 1 as the SNR approaches infinity.

\section{SyStem MOdeL}

A MIMO system with $N_{t}$ transmit antennas and $N_{r}$ receive antennas is considered. Let $M=\min \left(N_{t}, N_{r}\right)$ and $N=$ $\max \left(N_{t}, N_{r}\right)$. The channel between each pair of transmit and receive antennas is assumed to frequency flat. The received vector is given by:

$$
\mathbf{y}=\sqrt{P} \mathbf{H} \mathbf{x}+\mathbf{n},
$$

where $P$ is the average transmit power, $\mathbf{H}$ is the $N_{r} \times N_{t}$ matrix of channel coefficients, $\mathrm{x}$ is the $N_{t} \times 1$ transmitted 
vector, and $\mathbf{n} \sim \mathcal{C N}\left(0, \sigma_{n}^{2} I_{N_{r}}\right)$ is the additive white Gaussian noise (AWGN) vector. The entries of $\mathbf{H}$, i.e., $H_{i j}$, are i.i.d. circularly symmetric complex Gaussian with variance 0.5 per dimension. A correlated block fading channel model [5] is considered, where $\mathbf{H}$ is assumed to constant over a block of symbols (frame) and correlated across blocks (or frames).

\section{A. Imperfect CSI Model}

Channel estimation is performed using training symbols once in every frame. At the beginning of every frame, $N_{t}$ symbols are allocated for training, one for each transmit antenna. Minimum Mean Squared Error (MMSE) channel estimation is performed. The training symbol power can be increased compared to the data symbol power, without changing the average transmit power, to improve the quality of the estimate [11][12]. Assuming that the number of symbols in a frame is much larger than $N_{t}$, increasing the training power leads to a negligible change in the data power in order to maintain the same average transmit power. In FDD systems, there is a delay in feedback and the CSIT is delayed by one or more frames. In TDD systems, the channel estimate obtained from training the transmitter can be used for adaptation. Even in this case, there is a time delay between transmissions in the two directions. If the delay is known, channel prediction can be used to compensate for the delay.

Let $\mathbf{H}_{t}\left(N_{r} \times N_{t}\right.$ matrix) be the CSIT (used for adaptation) and $\mathbf{H}_{r}\left(N_{r} \times N_{t}\right.$ matrix) be the CSIR (used for decoding). For the Rayleigh fading model with AWGN, $\mathbf{H}_{t}$ and $\mathbf{H}_{r}$ are both zero mean and jointly Gaussian. Therefore, they can be related as follows:

$$
\mathbf{H}_{r}=\sigma_{r}\left[\frac{\rho}{\sigma_{t}} \mathbf{H}_{t}+\sqrt{1-\rho^{2}} \mathbf{E}\right]
$$

where $\mathbf{E} \sim \mathcal{C} \mathcal{N}(0, I), \sigma_{r}^{2}=E\left[\left|H_{r, i j}\right|^{2}\right], \sigma_{t}^{2}=E\left[\left|H_{t, i j}\right|^{2}\right]$, and $\rho=\frac{E\left[H_{r, i j} H_{t, i j}^{*}\right]^{r}}{\sqrt{E\left[\left|H_{r, i j}\right|^{2}\right] E\left[\left|H_{t, i j}\right|^{2}\right]}} . H_{r, i j}$ and $H_{t, i j}$ represent the $(i, j)^{t h}$ elements of the matrices $\mathbf{H}_{r}$ and $\mathbf{H}_{t}$ respectively. This imperfect CSI model can be used to model several scenarios. Some of these scenarios are discussed below.

Case 1: Estimation error, No feedback delay

In this case, $\mathbf{H}_{t}=\mathbf{H}_{r}=\hat{\mathbf{H}}$ (estimated CSI), $\rho=1$, and $\sigma_{t}^{2}=\sigma_{r}^{2}=\frac{P_{t}}{P_{t}+\sigma_{n}^{2}}=\left(1-\sigma_{e}^{2}\right)$, where $\sigma_{e}^{2}$ is the estimation error variance and $P_{t}$ is the transmit power used during training. Note that $\sigma_{e}^{2}$ decreases with increasing SNR.

Case 2: Feedback delay, No estimation error

In this case, $\mathbf{H}_{r}=\mathbf{H}$, and $\mathbf{H}_{t}=\mathbf{H}_{\text {old }}$ (past channel), $\sigma_{t}^{2}=\sigma_{r}^{2}=1$, and $\rho=J_{0}\left(2 \pi f_{d} T \Delta\right.$ ) (assuming Jakes' fading correlation model), where $J_{0}(x)$ is the zeroth order Bessel function, $f_{d}$ is the Doppler spread, $\Delta$ is the feedback delay in number of frames, and $T$ is the frame duration. Note that $\rho$ is independent of SNR and is strictly less than 1.

Case 3: Estimation error and Feedback delay

In this case, $\mathbf{H}_{r}=\hat{\mathbf{H}}$ and $\mathbf{H}_{t}=\hat{\mathbf{H}}_{\text {old }}, \sigma_{t}^{2}=\sigma_{r}^{2}=\frac{P_{t}}{P_{t}+\sigma_{n}^{2}}$, and $\rho=\frac{P_{t}}{P_{t}+\sigma_{n}^{2}} J_{0}\left(2 \pi f_{d} T \Delta\right)$. Note that $\rho<1$ and as $S N R \rightarrow \infty$, $\rho \rightarrow J_{0}\left(2 \pi f_{d} T \Delta\right)$.

Case 4: Estimation error, Feedback delay, and Prediction
An $L$-tap linear MMSE prediction filter is assumed. In this case, $\mathbf{H}_{r}=\hat{\mathbf{H}}$, and $\mathbf{H}_{t}=\tilde{\mathbf{H}}$ (predicted CSI). At time $k, \tilde{H}_{i j}(k)=\mathbf{w}^{H} \mathbf{h}_{i j, \Delta}(k)$, where $\mathbf{h}_{i j, \Delta}(k)=\left[\hat{H}_{i j}(k-\right.$ $\left.\Delta), \hat{H}_{i j}(k-\Delta-1), \cdots, \hat{H}_{i j}(k-\Delta-L+1)\right]^{T}$, and $\mathbf{w}$ is the vector of prediction filter coefficients. Since the channel between different pairs of transmit and receive antennas are i.i.d., the linear MMSE prediction filter coefficients are independent of $i$ and $j$ and given by $\mathbf{w}=\mathbf{R}^{-1} \mathbf{p}$, where $\mathbf{R}=E\left[\mathbf{h}_{i j, \Delta}(k) \mathbf{h}_{i j, \Delta}(k)^{H}\right]$ and $\mathbf{p}=E\left[H_{i j}(k) \mathbf{h}_{i j, \Delta}(k)^{H}\right]$. Further, it can be shown that $\sigma_{t}^{2}=\mathbf{p}^{H} \mathbf{w}=\mathbf{p}^{H} \mathbf{R}^{-1} \mathbf{p}$ and $\rho=\sqrt{\frac{P_{t}}{P_{t}+\sigma_{n}^{2}} \mathbf{p}^{H} \mathbf{R}^{-1} \mathbf{p}}$. Again, note that $\rho<1$ as $S N R \rightarrow \infty$ for a finite-tap predictor.

\section{B. Maximum eigen-mode beamforming}

Transmit beamforming is employed at the transmitter. The transmit vector at time $k$ is given by: $\mathbf{x}(k)=\mathbf{b} x(k)$, where $x(k)$ is the transmit symbol and $\mathbf{b}$ is the beamforming vector. In maximum eigen-mode beamforming, $\mathbf{b}$ is chosen to be $\mathbf{u}$, the right singular vector corresponding to the maximum singular value of $\mathbf{H}$. This choice of $\mathbf{b}$ maximizes $\|\mathbf{H b}\|^{2}$ and this maximum value of $\|\mathbf{H b}\|^{2}$ is $\lambda$, where $\sqrt{\lambda}$ is the maximum singular value. However, in practice, when imperfect CSI is available at the transmitter, $\mathbf{b}$ is chosen to be the right singular vector corresponding to the maximum singular value of $\mathbf{H}_{t}$.

\section{OUTAGE PROBABILITY ANALYSIS}

If the CSIT $\mathbf{H}_{t}$ is a delayed version of $\mathbf{H}_{r}$ obtained through a feedback channel from the receiver, the receiver knows $\mathbf{H}_{t}$ as well as $\mathbf{H}_{r}$ and the outage probability is:

$$
P(\text { outage })=P\left(I\left(x ; \mathbf{y} / \mathbf{H}_{t}, \mathbf{H}_{r}\right)<R\right),
$$

where $R$ is the rate of transmission. A lower bound on the mutual information between $x$ and $\mathbf{y}$, given $\mathbf{H}_{t}$ and $\mathbf{H}_{r}$, is given by $[11,12]$ :

$$
I\left(x ; \mathbf{H} / \mathbf{H}_{t}, \mathbf{H}_{r}\right) \geq \log \left[1+\Gamma \mathbf{u}^{H} \mathbf{H}_{r}^{H} \mathbf{H}_{r} \mathbf{u}\right],
$$

where $\Gamma=\frac{P}{P \sigma_{e}^{2}+\sigma_{n}^{2}}=\frac{S N R}{\left(\frac{P}{P_{t}+\sigma_{n}^{2}}\right)+1}$ and $S N R=\frac{P}{\sigma_{n}^{2}}$. Using this mutual information lower bound, an upper bound on outage probability can be obtained as:

$$
\begin{aligned}
P(\text { outage }) & \leq P\left(\log \left[1+\Gamma \mathbf{u}^{H} \mathbf{H}_{r}^{H} \mathbf{H}_{r} \mathbf{u}\right]<R\right) \\
& =P\left(\mathbf{u}^{H} \mathbf{H}_{r}^{H} \mathbf{H}_{r} \mathbf{u}<\beta\right),
\end{aligned}
$$

where $\beta=\frac{e^{R}-1}{\Gamma}$. Let $b=\mathbf{u}^{H} \mathbf{H}_{r}^{H} \mathbf{H}_{r} \mathbf{u}=\left\|\mathbf{H}_{r} \mathbf{u}\right\|^{2}$. Using equation (2), we get

$$
\begin{aligned}
b & =\sigma_{r}^{2}\left\|\frac{\rho}{\sigma_{t}} \mathbf{H}_{t} \mathbf{u}+\sqrt{1-\rho^{2}} \mathbf{E} \mathbf{u}\right\|^{2} \\
& =\frac{\left(1-\rho^{2}\right) \sigma_{r}^{2}}{2}\left\|\sqrt{\frac{2 \rho^{2}}{\left(1-\rho^{2}\right) \sigma_{t}^{2}}} \mathbf{H}_{t} \mathbf{u}+\sqrt{2} \mathbf{E} \mathbf{u}\right\|^{2}
\end{aligned}
$$

Let $A=\left\|\sqrt{\frac{2 \rho^{2}}{\left(1-\rho^{2}\right) \sigma_{t}^{2}}} \mathbf{H}_{t} \mathbf{u}+\sqrt{2} \mathbf{E} \mathbf{u}\right\|^{2}$. Given $\mathbf{H}_{t}, \mathbf{H}_{t} \mathbf{u}$ is a constant and $\sqrt{2} \mathbf{E u}$ is a $N \times 1$ vector of i.i.d zero 
mean complex Gaussian entries with variance 1 per dimension. Therefore, given $\mathbf{H}_{t}, A$ is noncentral chi squared distributed with $2 N$ degrees of freedom and noncentrality parameter $\delta=\frac{2 \mu}{\sigma_{t}^{2}}\left\|\mathbf{H}_{t} \mathbf{u}\right\|^{2}=\frac{2 \mu}{\sigma_{t}^{2}} \lambda$, where $\mu=\frac{\rho^{2}}{1-\rho^{2}}$. Therefore, we get

$$
\begin{aligned}
P\left(\text { outage } / \mathbf{H}_{t}\right) & \leq P\left(A<\frac{2(1+\mu) \beta}{\sigma_{r}^{2}}\right) \\
= & \sum_{j=0}^{\infty} \frac{e^{-\frac{\mu \lambda}{\sigma_{t}^{2}}}}{j !}\left(\frac{\mu \lambda}{\sigma_{t}^{2}}\right)^{j} \gamma_{j+N}\left(\frac{(1+\mu) \beta}{\sigma_{r}^{2}}\right)
\end{aligned}
$$

Equation (7) gives the upper bound on the conditional outage probability given $\lambda$, which can be averaged over $\lambda$ to get the upper bound on outage probability as:

$$
P(\text { outage }) \leq \int_{0}^{\infty} P_{U B}(\text { outage } / x) f_{\lambda}(x) d x .
$$

This upperbound can be simplified to the expression in equation (9) (see appendix for details) for rank 2 systems (i.e., $M=2$ ), where $\nu=\frac{\mu}{\sigma_{t}^{2}}$. This bound can be simplified to a closed-form expression using similar simplifications even when $M>2$. However, the expression is complicated and does not provide any further insight. Therefore, it is omitted.

In a TDD system, the receiver does not know $\mathbf{H}_{t}$. However, it estimates the product $\mathbf{H u}$. Even in this case, the bound in equation (5) can be used and the distribution of $A$ would remain the same given $\lambda$.

\section{A. Numerical Results}

The outage probability vs. SNR for a $2 \times 4$ system (evaluated using equation (9)) is shown in Fig 1 . The desired rate $R$ is 2 nats/sec/Hz. A normalized Doppler spread $f_{d} T=0.05$ (eg., $f_{d}=25 \mathrm{~Hz}, T=2 \mathrm{~ms}$ ) and delay $\Delta=1$ frame are considered, corresponding to a $\rho$ of 0.97 . The outage probability with perfect CSIT is also shown for reference. Fig. 1 illustrates that the effect of feedback delay becomes significant as SNR increases. The effect of estimation error is negligible at high SNR since the estimation error reduces with SNR. Using channel prediction compensates for the degradation due to delay. The length of the prediction filter required is discussed further in section III-B.

The outage probability is plotted vs. $R$ for a $2 \times 2$ system in Fig. 2 for SNR of $20 \mathrm{~dB}$. A normalized Doppler of 0.05 and delay $\Delta=2$ frames are considered. This plot can be used to determine the outage capacity for a given $P$ (outage). Outage capacity is relevant for systems that adapt the transmission rate based on feedback while maintaining a constant outage probability (or block error rate). For $P($ outage $)=0.01$, the outage capacity with delayed CSIT is about $12 \%$ lower than the outage capacity with prediction.

\section{B. Required value for correlation}

Channel estimation error is proportional to $S N R^{-1}$. Therefore, the effect of channel estimation error can usually be characterized by a simple SNR loss. However, the effect of feedback delay is different. If $\rho$ is constant with SNR, the effect of delay dominates at high SNR. In order to bound

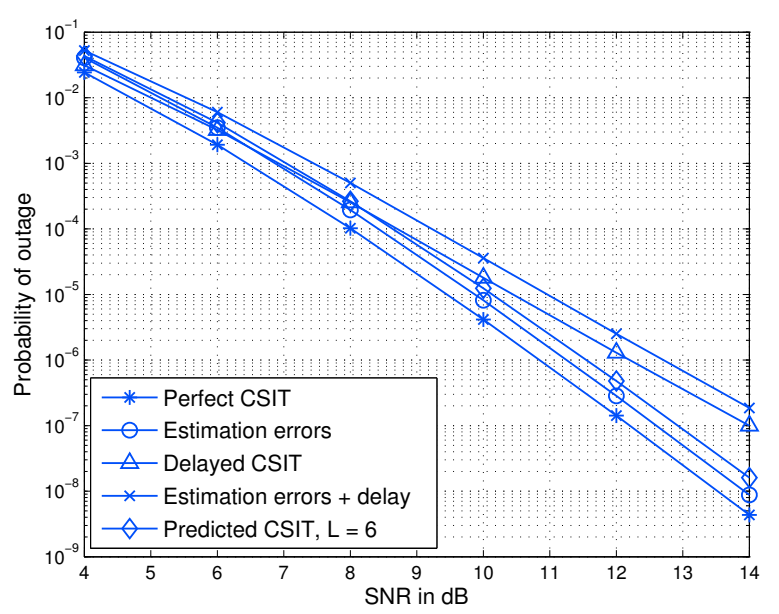

Fig. 1: Probability of outage vs. SNR for a $2 \times 4$ system, $R=2$ nats $/ \mathrm{s} / \mathrm{Hz}$, normalized Doppler $=0.05, \Delta=1$ frame.

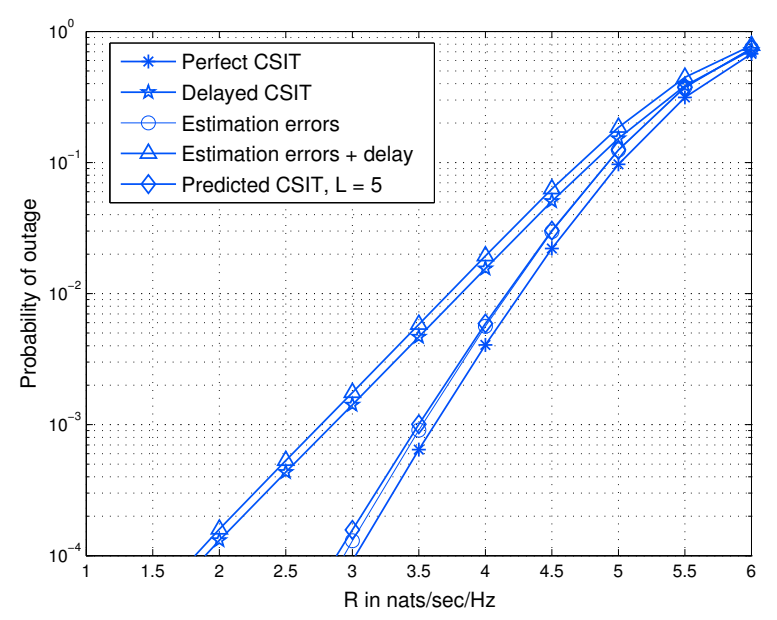

Fig. 2: Probability of outage vs. $\mathrm{R}$ for $2 \times 2, \mathrm{SNR}=20 \mathrm{~dB}$, normalized Doppler $=0.05, \Delta=2$ frames.

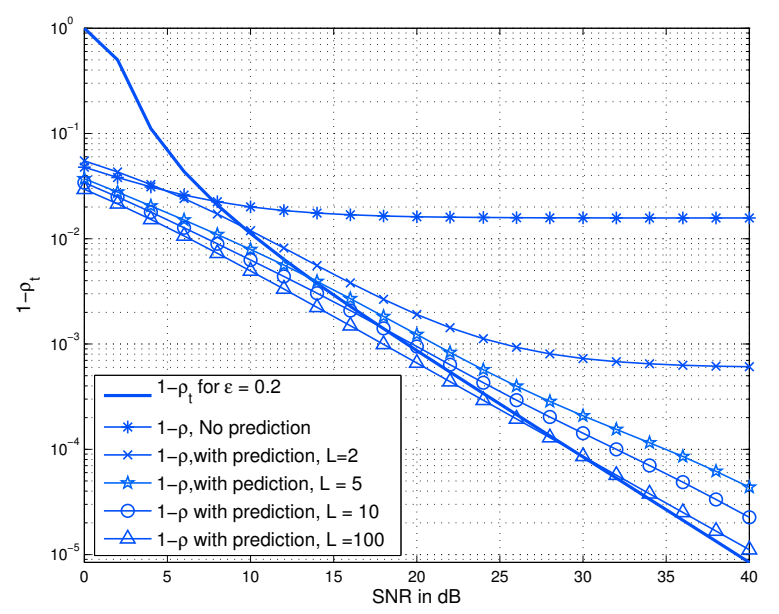

Fig. 3: $1-\rho_{t}$ vs. SNR for $2 \times 2$, normalized Doppler $=0.05, \Delta=1$. 


$$
\begin{aligned}
& P(\text { outage }) \leq \frac{1}{(N-1) !(N-2) !}\left[\frac{N !(N-2) !}{1+\nu} \sum_{k=0}^{1}\left(\begin{array}{l}
1 \\
k
\end{array}\right) \nu^{k} \gamma_{k+N}\left(\beta\left(\frac{1+\mu}{1+\nu}\right)\right)-2((N-1) !)^{2} \gamma_{N}\left(\beta\left(\frac{1+\mu}{1+\nu}\right)\right)+\right. \\
& \frac{N !(N-2) !}{(-\nu)^{N-1}} \frac{2+\nu}{2} \sum_{l=0}^{N-2}\left(\frac{-\nu}{2}\right)^{l} \gamma_{l+1}\left(2 \beta \frac{1+\mu}{2+\nu}\right)-\frac{N !(N-2) !}{(-\nu)^{N-1}}(1+\nu) \sum_{l=0}^{N-2}(-\nu)^{l} \gamma_{l+1}\left(\beta\left(\frac{1+\mu}{1+\nu}\right)\right)- \\
& \left.\frac{1}{2^{N}} \sum_{l=-1}^{N-4}\left[\frac{(N-2) !}{l !}+\frac{N !}{(l+2) !}-2 \frac{(N-1) !}{(l+1) !}\right](N+l) !\left(\frac{1}{2+\nu}\right)^{l+1} \sum_{k=0}^{l+1}\left(\begin{array}{c}
l+1 \\
k
\end{array}\right)\left(\frac{\nu}{2}\right)^{k} \gamma_{N+k}\left(2 \beta \frac{1+\mu}{2+\nu}\right)\right],
\end{aligned}
$$

the effect of delay by an SNR loss, the value of $\rho$ needs to increase with SNR and approach 1 as SNR ends to infinity. We

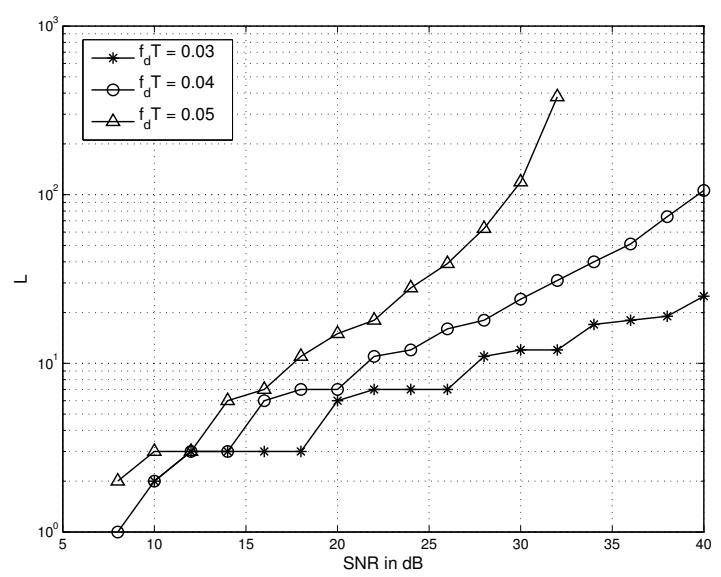

Fig. 4: Required prediction filter length $\mathrm{L}$ vs. SNR for $2 \times 2$ system, normalized Doppler $=0.05, \Delta=1$.

now numerically evaluate the minimum value of $\rho$ required, defined as $\rho_{t}$, such that the outage probability with delay is bounded by $(1+\epsilon)$ times the outage probability without delay. $\epsilon$ can be chosen depending on the acceptable degradation in performance. Fig. 3 shows the value of $1-\rho_{t}$ for a $2 \times 2$ system for $R=2$ nats $/ \mathrm{sec} / \mathrm{Hz}$ and $\epsilon=0.2$. The result indicates that $1-\rho_{t} \propto S N R^{-1}$ at high SNR. The value of $\rho$ with $L$-tap prediction filters is also shown for $L=2,5,10$ and 100. It can be seen that prediction is not necessary up to the SNR of $7 \mathrm{~dB}$. $L=10$ and 100 limit degradation up to SNR of $25 \mathrm{~dB}$ and $40 \mathrm{~dB}$ respectively. Fig. 4 depicts the prediction filter length required to achieve the $\rho_{t}$ for various normalized Doppler frequencies. At SNR of $30 \mathrm{~dB}, \mathrm{~L}=10,25$ and 100 are required for $\Delta=1$ and $f_{d} T=0.03,0.04$ and 0.05 respectively.

\section{ASYMPTOTIC DIVERSITY ORDER}

In this section, we analyze the asymptotic diversitymultiplexing-feedback quality tradeoff for MIMO maximum eigen-mode beamforming. Feedback quality $\alpha$ is defined as the rate at which $\rho \rightarrow 1$ as $S N R \rightarrow \infty$, i.e.,

$$
\alpha=-\frac{\log (1-\rho)}{\log S N R} \text {. }
$$

The diversity $d$ and multiplexing gain $r$ are defined as in [13]. With perfect CSIT, $d=N_{t} N_{r}(1-r)$.
Theorem: The asymptotic diversity gain $d$ of maximum eigen-mode beamforming with imperfect CSI is given by:

$$
d=\left\{\begin{array}{ll}
N_{r}\left(\alpha\left(N_{t}-1\right)+1-r\right) & \text { for } \alpha<(1-r) \\
N_{t} N_{r}(1-r) & \text { for } \alpha>(1-r)
\end{array},\right.
$$

for $0 \leq \alpha \leq 1$ and $0 \leq r \leq 1$.

Proof: See appendix.

Note that if $1-\rho \propto S N R^{-1}$, i.e., $\alpha=1$, the diversity order

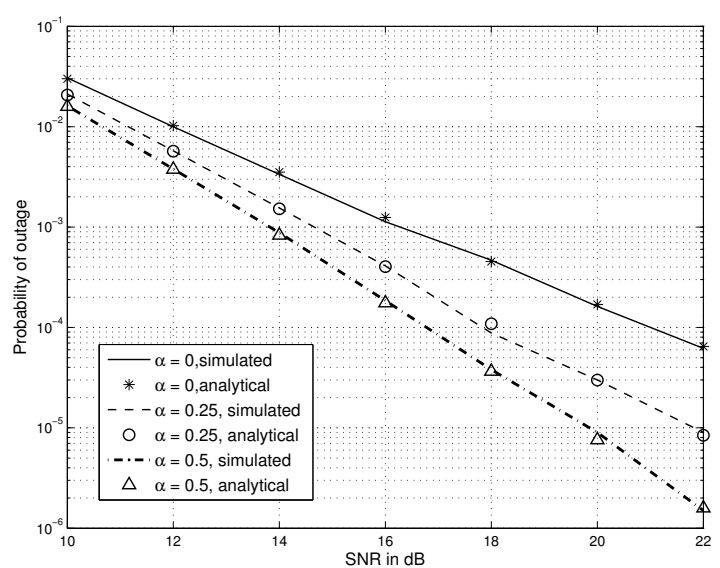

Fig. 5: Probability of outage vs. SNR for $2 \times 2$ system for different values of $\alpha$

is the same as the perfect CSI case. The analytical results for a $2 \times 2$ system for $\alpha=0, \alpha=0.25$ and $\alpha=0.5$ are also compared with Monte Carlo simulation results in Fig. 5. The desired rate $R$ is 2 nats $/ \mathrm{sec} / \mathrm{Hz}$. It can be seen that the asymptotic diversity gain is 2 for $\alpha=0,2.5$ for $\alpha=0.25$ and 3 for $\alpha=0.5$ validating the analysis.

\section{Conclusions}

The effect of feedback delay on the outage probability and outage capacity of maximum eigen-mode beamforming system is analyzed. The usefulness of channel prediction in combating the effect of feedback delay is also studied. The outage probability of a system operating at fixed rate is shown to degrade because of delayed feedback. For rate adaptive systems that maintain a fixed $P$ (outage), delayed feedback results in lower outage capacity. Evaluation of the minimum $\rho$ required to limit degradation indicates that $1-\rho$ should be proportional to $\mathrm{SNR}^{-1}$. The length of the prediction filter required to attain the required value of $\rho$ is also calculated. 
Since the required length increases with SNR and can be large at high SNR, employing channel prediction to improve the performance is practical mainly at low and moderate SNRs. The asymptotic diversity order analysis shows that if $1-\rho \propto S N R^{-1}$, the diversity order is unaltered, validating the evaluation of $\rho_{t}$.

\section{ACKNOWLEDGMENT}

This work was supported in part by the Dept. of Science and Technology, Govt. of India.

\section{APPENDIX}

Derivation of equation (9): $P$ (outage) is upper bounded by

$$
\sum_{j=0}^{\infty} \frac{\gamma_{j+N}\left(\beta \frac{1+\mu}{\sigma_{r}^{2}}\right)}{j !} \int_{0}^{\infty} e^{-\frac{\mu}{\sigma_{t}^{2}} x}\left(\frac{\mu}{\sigma_{t}^{2}} x\right)^{j} f_{\lambda}(x) d x
$$

For rank 2 channels, the pdf of $\lambda$ is can be written as [14]:

$$
\begin{aligned}
& f_{\lambda}(x)=c e^{-x} x^{N-2}\left[N !-2(N-1) ! x+(N-2) ! x^{2}\right]- \\
& e^{-2 x} \sum_{k=-2}^{N-4}\left[\frac{(N-2) !}{k !}+\frac{N !}{(k+2) !}-2 \frac{(N-1) !}{(k+1) !}\right] x^{N+k}
\end{aligned}
$$

where $c=\frac{1}{(N-1) !(N-2) !}$. Simplifying equation (12), reduces to evaluating the following expression

$$
\sum_{j=0}^{\infty} \frac{\gamma_{j+N}\left(\beta \frac{1+\mu}{\sigma_{r}^{2}}\right)}{j !} \int_{0}^{\infty} e^{-x \frac{\mu}{\sigma_{t}^{2}}}\left(\frac{\mu x}{\sigma_{t}^{2}}\right)^{j} e^{-m x} x^{l} d x
$$

for different values of $l$ and $m$ and adding them. For $l>=$ $N-1$, this expression simplifies to

$$
\frac{l !}{m^{N}} \frac{1}{(\nu+m)^{l+1-N}} \sum_{p=0}^{l+1-N}\left(\frac{\nu}{m}\right)^{p} \gamma_{N+p}\left(m \beta \frac{1+\mu}{m+\nu}\right),
$$

and for $l=N-2$, it simplifies to

$$
\begin{aligned}
& \frac{(N-2) !}{(-\nu)^{N-1}}\left[\gamma_{1}((1+\mu) \beta)-\sum_{p=0}^{N-2}\left(-\frac{\nu}{m}\right)^{p}\right. \\
& \left.\left(\frac{\nu+m}{m}\right) \gamma_{p+1}\left(m \beta \frac{1+\mu}{m+\nu}\right)\right] .
\end{aligned}
$$

Proof of Theorem: This proof closely follows the analysis method in [15]. First, we split the integration interval over $\lambda$ into two intervals $[0, B]$ and $[B, \infty)$, where $B$ is chosen such that $f_{\lambda}(x)=c \frac{x^{p-1}}{(p-1) !}+o\left(x^{p}\right)$ in the interval $[0, B]$ and $p=N_{r} N_{t}$. Now, the outage probability can be expressed as

$$
P(\text { outage })=T_{1}-T_{2}+T_{3},
$$

where $T_{1}=c \int_{0}^{\infty} P($ outage $/ x) \frac{x^{p-1}}{(p-1) !} d x, T_{2}=\int_{B}^{\infty}$ $P($ outage $/ x) c \frac{x^{p-1}}{(p-1) !} d x$ and $T_{3}=\int_{B}^{\infty} P($ outage $/ x) f_{\lambda}(x) d x$. $T_{1}-T_{2}$ corresponds to the integral over $[0, B]$ and $T_{3}$ corresponds to the integral over the other region.

$T_{1}$ simplifies similar to the expression in equation (14) to

$$
T_{1}=c\left(\frac{1}{\mu}\right)^{p} \sum_{j=0}^{\infty} \frac{(j+p-1) !}{j !(p-1) !} \gamma_{j+N_{r}}((1+\mu) \beta)
$$

Writing the Gamma function as an integral and interchanging the order of summation and integration results in:

$$
T_{1}=c\left(\frac{1}{\mu}\right) \sum_{k=0}^{p}\left(\begin{array}{c}
p-N_{r} \\
k
\end{array}\right) \frac{((1+\mu) \beta)^{N_{r}+k}}{\left(N_{r}+k\right) !} .
$$

As $S N R \rightarrow \infty, \beta \approx\left(\frac{1}{S N R}\right)^{1-r}$ and $\mu \approx S N R^{\alpha}$ Therefore, $T_{1}$ has diversity same as equation (11). Then, we can show that $T_{2}$ and $T_{3}$ decay faster than $T_{1}$ with SNR in order to prove the result in the theorem as in [15]. In order to do this, $T_{2}$ is written as:

$$
T_{2}=c \sum_{j=0}^{\infty} \frac{\gamma_{j+N_{r}}((1+\mu) \beta)}{j !(p-1) !}\left(\frac{1}{\mu}\right)^{p} \int_{\mu B}^{\infty} e^{-y} y^{j+p-1} d y,
$$

where $y=\mu x$. As $S N R \rightarrow \infty, \mu \approx S N R^{\alpha}$, and the integral above approaches $0 . T_{3}$ is bounded as (where $y=\mu x$ ):

$$
T_{3} \leq c e^{-\mu B} \sum_{j=0}^{\infty} \frac{\gamma_{j+N_{r}}((1+\mu) \beta)}{j} \int_{\mu B}^{\infty} e^{-y}(y)^{j} f_{\lambda}(y) d y .
$$

\section{REFERENCES}

[1] E. Dahlmann, S. Parkvall, J. Skold, P. Beming, "3G Evolution: HSPA and LTE for Mobile Broadband," Academic Press, Second Edition, 2008.

[2] S. Zhou, G. B. Giannakis, "Optimal transmitter eigen-beamforming and space-time block coding based on channel mean feedback," IEEE Trans. on Sig. Proc., vol.50, no.10, pp. 2599-2613, Oct 2002.

[3] S. Zhou, G. B. Giannakis, "How accurate channel prediction needs to be for transmit-beamforming with adaptive modulation over Rayleigh MIMO channels?," IEEE Trans. on Wireless Comm., vol.3, no.4, pp. 1285-1294, July 2004

[4] E. N. Onggosanusi, A. Gatherer, A. G. Dabak, S. Hosur, "Performance analysis of closed-loop transmit diversity in the presence of feedback delay," IEEE Trans. on Comm., vol.49, no.9, pp.1618-1630, Sep 2001

[5] V. S. R. Annapureddy, S. Bhashyam, "Spatial and temporal power allocation for MISO systems with delayed feedback," Proceedings of 40th Asilomar conference on Signals, Systems, and Computers, Pacific Grove, CA, Oct-Nov. 2006.

[6] K. Huang, B. Mondal, R. W. Heath, J. G. Andrews, 'Effect of Feedback Delay on Multi-Antenna Limited Feedback for Temporally-Correlated Channels," IEEE GLOBECOM 2006, pp.1-5, Nov-Dec 2006.

[7] G. Lebrun, J. Gao, M. Faulkner, "MIMO transmission over a timevarying channel using SVD," IEEE Trans. on Wireless Comm., vol.4, no.2, pp. 757-764, March 2005.

[8] K. Kobayashi, T. Ohtsuki, T. Kaneko, "MIMO Systems in the Presence of Feedback Delay," IEEE ICC 2006, vol.9, pp.4102-4106, June 2006.

[9] E. K. S. Au, W. H. Mow, "Exact bit error rate for SVD-based MIMO systems with channel estimation errors," Proc. IEEE ISIT 2006, pp. 2289-2293, July 2006.

[10] E. K. S. Au, S. Jin, M. R. McKay, W. H. Mow, X. Gao, I. B. Collings, "Analytical Performance of MIMO-SVD systems in Ricean fading channels with channel estimation error and feedback delay," IEEE Trans. on Wireless Comm., pp. 1315-1325, vol. 7,No. 4, Apr 2008.

[11] B. Hassibi, B. M. Hochwald, "How much training is needed in multiple antenna wireless links?," IEEE Trans. on Info. Theory, vol. 49, no. 4, pp. 951-963, Apr. 2003.

[12] S. Bhashyam, A. Sabharwal, B. Aazhang, "Feedback gain in multiple antenna systems", IEEE Trans. on Comm., vol. 50, no. 5, pp.785-798, May2002.

[13] L. Zheng, D. N. C. Tse, "Diversity and multiplexing: a fundamental tradeoff in multiple-antenna channels," IEEE Trans. on Info. Theory, vol.49, no.5, pp. 1073-1096, May 2003.

[14] I. E. Telatar, "Capacity of multi-antenna Gaussian channels," Europ. Trans. Telecommu., vol. 10, pp. 585-595, Nov./Dec. 1999.

[15] Z. Wang, G. B. Giannakis, "A simple and General Parametrization Quantifying Performance in Fading Channels," IEEE Trans. on Comm., vol. 51, No. 8, Aug. 2003. 